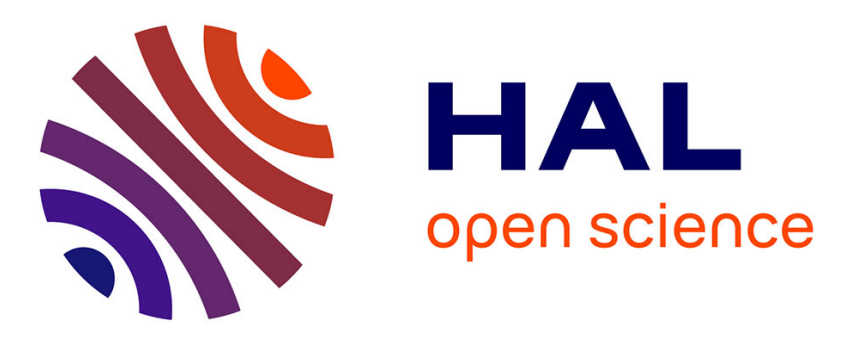

\title{
Controlling Confinement and Topology to Study Collective Cell Behaviors
}

\author{
Guillaume Duclos, Maxime Deforet, Hannah G Yevick, Olivier \\ Cochet-Escartin, Flora Ascione, Sarah Moitrier, Trinish Sarkar, Victor \\ Yashunsky, Isabelle Bonnet, Axel Buguin, et al.
}

\section{To cite this version:}

Guillaume Duclos, Maxime Deforet, Hannah G Yevick, Olivier Cochet-Escartin, Flora Ascione, et al.. Controlling Confinement and Topology to Study Collective Cell Behaviors. Cell Migration, pp.387-399, 2018, Methods in Molecular Biology, 10.1007/978-1-4939-7701-7_28 . hal-02063376

\section{HAL Id: hal-02063376 https://hal.science/hal-02063376}

Submitted on 11 Mar 2019

HAL is a multi-disciplinary open access archive for the deposit and dissemination of scientific research documents, whether they are published or not. The documents may come from teaching and research institutions in France or abroad, or from public or private research centers.
L'archive ouverte pluridisciplinaire HAL, est destinée au dépôt et à la diffusion de documents scientifiques de niveau recherche, publiés ou non, émanant des établissements d'enseignement et de recherche français ou étrangers, des laboratoires publics ou privés. 


\section{Controlling confinement and topology to}

\section{study collective cell behaviors}

G. Duclos ${ }^{+}$, M. Deforet ${ }^{+}$, H.G. Yevick ${ }^{+}$, O. Cochet-Escartin ${ }^{+}$, F. Ascione, S. Moitrier, T. Sarkar, V. Yashunsky, I. Bonnet, A. Buguin, P. Silberzan*

Laboratoire PhysicoChimie Curie, Institut Curie, PSL Research University - Sorbonne Universités, UPMC - CNRS - Equipe labellisée Ligue Contre le Cancer ; 75005, Paris, France.

${ }^{+}$Equal contribution
* pascal.silberzan@curie.fr

Running title: Control of cell monolayer confinement and topology 


\section{$\underline{\text { Abstract }}$}

Confinement and substrate topology strongly affect the behavior of cell populations and, in particular, their collective migration. In vitro experiments dealing with these aspects require strategies of surface patterning that remain effective over long times (typically several days) and ways to control the surface topology in 3 dimensions. Here we describe protocols addressing these two aspects. High-resolution patterning of a robust cell-repellent coating is achieved by etching the coating through a photoresist mask patterned directly on the coated surface. Out-of-plane curvature can be controlled using glass wires or corrugated 'wavy' surfaces.

Keywords: Collective cell migration, Microfabrication, Surface patterning, Out-of-plane curvature, Positive curvature, Negative curvature 


\section{Introduction}

Collective cell migration has been identified as a major mode of cell migration in vivo and can be recapitulated in vitro using for instance wound healing experiments [1-5]. In vivo, collective migrations are often accompanied by physical confinement (cells migrating between vessels or capillaries or onto such structures [5]). Therefore, in vitro experiments aimed at mimicking these biologically relevant situations often include such a confinement, usually by constraining the cells within chemically-defined patterns such as stripes in a planar geometry [6] or in other topologies including the third dimension [79]. Two-dimensional tracks, onto which cells adhere, are defined within an anti-adhesive substrate. Practically, requirements for the robustness of the anti-adhesive coating are particularly stringent as the experiments can last days or even weeks, whereas most of the classical cell-repellent coatings are based on physisorbed PolyEthyleneGlycol (PEG)-based copolymers and are only stable for a couple of days at best.

Here, we present two technologies that combine the coating of the substrate using a robust anti-adhesive polymer layer with high-resolution photolithography. The Aam-PEG anti-adhesive coating is adapted from Reference [10]. It consists in successive grafts on a glass coverslip: Silane molecules covalently bind to clean glass and form a reactive layer to which a thin layer of polyacrylamide gel can be anchored; finally, PEG-acrylamide molecules interpenetrate the gel and crosslink it to form a dense hydrophilic brush that prevents cell adhesion. The resulting coating is strongly repellent to the cells and extremely robust (up to a couple of months) $[\mathbf{1 1}, \mathbf{1 2}]$. The Aam-PEG coating is compatible with photolithography, it can be patterned at the $\mu \mathrm{m}$-scale resolution by plasma etching though a photoresist mask patterned directly onto the PEG coating using standard photolithography techniques [12-14]. It can also be patterned using the deep-UV etching technique where the substrate is exposed to deep UV radiation through a mask [15-17]. This second method is faster than the previous one and avoids photolithography steps. Both methods allow for a strong confinement of cell populations for weeks, after the cells have reached confluence on the pattern. 
In vivo, cells are, of course, not constrained to 2D space. Tissues routinely form complex 3D structures, many of which being highly curved. For example, cells that migrate out of tumors often wrap around vessels or extracellular matrix fibers and migrate effectively on a curved cylindrical substrate [5]. We describe a method to make model glass fibers that can be used as substrates to study collective cell migration. Their radius can be varied from $\sim 100 \mu \mathrm{m}$ down to submicron values [7]. Corrugated 'wavy' surfaces represent an excellent assay for probing how cells migrate, orient and respond to positive and negative out-of-plane curvatures. This method requires a castellated PolyDiMethylSiloxane (PDMS) substrate produced by thick resist photolithography that is embossed into molten polystyrene (PS). The resultant PS castellated structure is then reflowed to achieve a well-controlled curved wavy substrate that can be replicated at will by molding PDMS for cell culture.

We often use glass as our final substrate for cell culture. We find that this is an adequate substrate for most purposes. Cells adhere on untreated glass probably via the adsorption of proteins present in the culture medium. However, we also provide protocols for fibronectin coating of the patterned substrates. When coated with fibronectin, cell dynamics is usually faster compared to bare glass.

After preparation, cells of interest are seeded on these various substrates and observed by optical microscopy at all relevant scales, using the most adequate modes (live imaging coupled to time-lapse or confocal imaging on fixed cells in particular when dealing with out-of-plane curvature). The role of collective migration is not only crucial for cells bearing stable cell-cell adhesions such as epithelial cells [3], but also for other types of cells such as elongated fibroblasts $[\mathbf{1 4}, \mathbf{1 8}]$.

\section{Material}

Prepare all aqueous solution with deionized (DI) water. Use analytical grade reactants and solvents. Dispose chemical waste appropriately.

\subsection{Aam-PEG Coating}

1. Glass coverslips (typical diameter 30mm). 
2. "Piranha" solution: 1 vol concentrated sulfuric acid / 3 vol 30\%-hydrogen peroxide solution (see Notes 1 and 14).

3. Silane solution : $200 \mu \mathrm{L}$ of Allyltrichlorosilane (Sigma) in $15.5 \mathrm{~mL}$ Toluene (see Note 1).

4. Acrylamide solution: $3 \mathrm{~g}$ Acrylamide (Sigma), $0.3 \mathrm{~g} \mathrm{~N}, \mathrm{~N}^{\prime}-M e t h y l e n e b i s(a c r y l a m i d e)$ (BIS) (Sigma), 1.09g Benzophenone (Sigma) in 20mL Acetone (see Notes 1 and 16).

5. PEG solution : mix 0.6 g Poly(ethylene glycol) methyl ether methacrylate (PEG) (Sigma) (see Note 2), $0.9 \mathrm{~g}$ BIS, 1.09g Benzophenone in 20mL Methanol (see Note 16).

6. Hot plate.

7. UV ( $\lambda=365 \mathrm{~nm}) 25 \mathrm{~W}$-Transilluminator (UVP, TFL 40V) (see Note 3 ).

8. Air Plasma (Harrick Plasma cleaner).

\subsection{Photolithography-based patterning of PEG coating}

If possible, use a clean room equipped with standard photolithography equipment (necessary for small features). If high-resolution is not an issue, a clean environment may be sufficient.

1. Spin coater and mask aligner such as a MJB3 mask aligner (Karl Suss) (see Note 4). Hot plate.

2. Chrome mask with the desired pattern (glass or quartz) (Compugraphics, UK) (see Note 5).

3. S1813 Positive Photoresist (Shipley).

4. 351 Developer (Shipley) diluted in DI water (1v/4v).

5. $10 \mu \mathrm{g} / \mathrm{mL}$ Fibronectin solution in PBS.

6. Acetone.

7. Air Plasma (Harrick plasma cleaner).

\subsection{Deep-UV patterning of PEG coating}

1. Chrome mask with the desired pattern (Synthetic quartz quality (see Note 6), Compugraphics, UK) (see Note 5).

2. Deep-UV lamp, $\lambda=185 \mathrm{~nm}$ (UVO Cleaner, Jelight, USA) (see Note 7 and 8)

3. $10 \mu \mathrm{g} / \mathrm{mL}$ Fibronectin solution in PBS 


\section{PBS}

5. PDMS mix: mix the two parts of the Sylgard 184 kit (Dow Corning) (1v/10v) (see Note 1)

6. Parafilm

7. Teflon tweezers (Sigma)

8. Glass bottom 6 well plate (IBL) or plastic bottom 6-well plate whose polystyrene bottom has been removed by drilling.

\subsection{Wire substrates}

1. Glass Pasteur pipettes (Fisher Scientific) (see Note 9) or glass rods (WPI Inc, diameter 1mm) (see Note 10)

2. PDMS films (thickness 17 mils) (Gel-Pak, Hayward)

3. PDMS mix: mix the two parts of the Sylgard 184 kit (Dow Corning) (1v/10v) (see Note 1)

4. $70 \%$ Ethanol

5. $10 \mu \mathrm{g} / \mathrm{mL}$ Fibronectin solution in PBS

6. $\mathrm{PBS}$

7. $0.1 \mathrm{mg} / \mathrm{mL}$ solution of Poly L-lysine-PolyEthylene Glycol (PLL(20)-g-[3,5]-PEG(2)) (Susos, Switzerland) [16]

8. Razor blade

9. Tweezers

10. Glass coverslips $(22 \times 30)$

11. Glass bottom 6-well plate (IBL GmbH, Germany)

12. Air Plasma cleaner (Harrick Plasma)

13. Pipette puller (P-2000, Sutter Instruments) (see Note 11)

\subsection{Wavy Substrates}

1. Chrome mask patterned with a periodic succession of chrome lines (width $\mathrm{w}$, period $\mathrm{p}$ ) (Compugraphics, UK) (see Notes 12 and 13) 
2. SU8-2150 photoresist (Microchem)

3. SU8 developer (Microchem)

4. Fluorosilane (tridecafluoro-1,1,2,2 tetrahydrooctyl trichlorosilane) (Roth Sochiel)

5. $10 \mu \mathrm{g} / \mathrm{mL}$ Fibronectin solution in PBS

6. Polystyrene polymer (granules) (Sigma)

7. PDMS mix: mix the two parts of the Sylgard 184 kit (Dow Corning) (1v/10v) (see Note 1)

8. Air Plasma cleaner (Harrick Plasma)

9. Heating Press (E-Z press, ICL)

10. Vacuum Oven (Precision)

\section{Methods:}

\subsection{Aam-PEG Coating}

1. Plasma clean the coverslips for $5 \mathrm{~min}$.

2. Prepare the piranha solution (see Note 14). Add the coverslips and let stand for $5 \mathrm{~min}$.

3. Rinse the coverslips with DI water. Dry under a jet of air. Put the dried coverslips in a clean glass Petri dish.

4. Prepare the allyltrichlorosilane solution. Pour the solution onto the clean coverslips and let stand for 5 min. Rinse with Toluene for $1 \mathrm{~min}$. Then, rinse with Acetone followed by DI water. Dry under a jet of air (see Note 15). Bake the silanized coverslips in a glass Petri dish on a hot plate $\left(90^{\circ} \mathrm{C}\right)$ for $1 \mathrm{~h}$.

5. Prepare the acrylamide solution (see Note 16). Put the freshly prepared acrylamide solution in a glass Petri dish and place the silanized coverslips in the dish (see Note 17). Let stand for 5 min. Place the dish on the transilluminator and expose the slides in the solution for 3 min (see Note 18) through the glass bottom of the dish (see Notes 3,19 and 20). The side of the coverslips facing the illuminator are now acrylamide-coated.

6. Rinse each slide individually with Acetone and then DI water. Dry the coverslips under a jet of air (see Notes 21 and 22). 
7. Prepare the PEG solution and place it in a glass bottom Petri dish. Incubate the acrylamidecoated coverslips in the solution, grafted side facing down, for 5 min (see Note 17). Expose the slides in the solution through the glass bottom of the dish with the transilluminator for $3 \mathrm{~min}$ (see Notes 3 and 23).

8. Rinse each slide individually with Methanol, then Acetone, and finally DI water. Dry the coverslips under a jet of air. At this point, the coated coverslips can be stored in a dust free environment for several months.

\section{2: Photolithography-based patterning of PEG coating (Fig. 1A)}

1. Spincoat S1813 positive photoresist (4000 rpm, $30 \mathrm{~s}$ ) on the coverslips prepared with the cell repellent coating as per section 3.1 (see Note 24). Softbake the slides: $115^{\circ} \mathrm{C}, 2 \mathrm{~min}$ on the hot plate (see Note 25).

2. If necessary, clean the mask with isopropanol.

3. Expose the coverslips through the mask (UV light, $\lambda=365 \mathrm{~nm}$ ) with the mask aligner (see Note 26).

4. Develop the exposed slides in Developer solution for $10 \mathrm{~s}$.

5. Rinse with DI water. Dry under a jet of air.

6. Etch the unprotected repellent layer with 4 runs of 5 min each, using a low power air plasma (see Note 27).

7. Optional: Fibronectin coating: Pipette $50 \mu \mathrm{L}$ of fibronectin solution on a parafilm layer. Return the coverslip on to the fibronectin drop so the patterned side is in direct contact with the fibronectin solution. Incubate for $1 \mathrm{~h}$.

8. Wash the coverslips with acetone to remove the protective layer of photoresist (see Note 28). Rinse with DI water and dry under a jet of air. The glass substrate is now patterned with cellrepellent areas and glass or fibronectin-coated areas. 
9. Incubate the cells at appropriate temperature and humidity for $2 \mathrm{~h}$ (see Note 29), to allow them to adhere to the adhesive parts of the substrate.

10. Aspirate medium with unattached cells. Wash with warm PBS and fill with warm culture medium. Cells can now be grown and observed. (Fig. 2A).

\subsection{Deep-UV patterning of PEG coating (Fig. 1A)}

1. Prepare the coverslips with the repellant coating as in section 3.1 (see Note 30).

2. Wash the quartz mask with Ethanol. Expose the mask to deep-UV light, Chrome side facing the lamp (see Note 31) for extensive cleaning.

3. Pipette 5 to $10 \mu \mathrm{L}$ of DI water onto the Chrome side of the mask (see Note 31) and return the coverslip onto the drop so the Aam-PEG coating faces the quartz mask and holds to it by capillary action (see Note 32).

4. Expose the coated coverslip through the mask for 15 min with the deep UV lamp.

5. Gently detach the coverslip from the mask using Teflon tweezers (see Note 33).

6. Rinse the coverslip with DI water and dry under a jet of air.

7. Fibronectin coating of the etched areas: Pipette $50 \mu \mathrm{L}$ of fibronectin solution on a parafilm layer. Return the coverslip on to the fibronectin drop so the patterned side is in direct contact with the fibronectin. Incubate for 1 hour. Rinse the coverslip with PBS, DI water and dry under a jet of air. The coverslips are now patterned.

8. If needed, attach the coverslip to the bottom of a 6-well plate whose bottom has been previously removed by drilling, using the PDMS mix. In that case, as fibronectin denaturates above $60^{\circ} \mathrm{C}$, cure PDMS overnight in a $37^{\circ} \mathrm{C}$ incubator.

9. Incubate the cells at appropriate temperature and humidity for $2 \mathrm{~h}$ to allow them to adhere to the substrate.

10. Aspirate medium with unattached cells. Wash with warm PBS and fill wells with warm culture medium. Cells can now be grown and observed. 


\subsection{Glass wires}

Glass capillaries of radius larger than $20 \mu \mathrm{m}$ are manufactured by heating glass Pasteur over the flame of a freestanding gas torch and pulling each end (see Note 34) [7]. Glass capillaries whose radius is lower than $20 \mu \mathrm{m}$ are manufactured from glass rods, using a pipette puller. Glass capillaries of a few fixed sizes can also be purchased (eg. $\mathrm{r}=40 \mu \mathrm{m}, 60 \mu \mathrm{m}, 85 \mu \mathrm{m}, \mathrm{CM}$ Scientific). This section describes how to assemble these wires into a device which can be used for migration experiments.

1. Sort wires by size under a stereomicroscope and set aside sizes of interest.

2. Cut PDMS Gel-Pak film into a rectangle $(\sim 0.5 \mathrm{~cm} \times 1.5 \mathrm{~cm})$ using the razor blade and peel from its polyester backing and polyethylene release liner.

3. Place the rectangular piece of Gel-Pak on a coverslip

4. Cut glass wires to size and align them parallel on the Gel-Pak protruding off the support on both end.

5. Cover carefully the Gel-Pak with the freshly prepared PDMS mix to immobilize the wires. Use only a minimal amount to avoid the PDMS from coating the freely suspended wires via capillary action.

6. Cure the structure for $24 \mathrm{~h}$ at $65^{\circ} \mathrm{C}$.

7. Clean the structures with ethanol and DI water. Air dry under a jet of air.

8. Expose the structures to an air plasma for $30 \mathrm{~s}$ to activate the PDMS and the glass wires.

9. Optional: Fibronectin coating: Incubate the wires in fibronectin solution for $1 \mathrm{~h}$. Aspirate the Fibronectin solution. Rinse the structures with PBS and dry under a jet of air for $5 \mathrm{~min}$.

10. Pre-treat the glass bottom of the 6-well plates with PLL-PEG solution $(1 \mathrm{~h})$ to prevent cells from growing down and off the structures onto the dish in the optical path (clouding the imaging of the wires)

11. Transfer the wire substrates to a 6-well plate with a tweezer.

12. Passage cells to obtain a cell suspension of the desired concentration (see Note 35).

13. Deposit a small drop of the cell suspension on PDMS pedestals $(\sim 50-100 \mu \mathrm{L}$ depending on PDMS substrate area). 
14. Incubate the substrates at appropriate temperature and humidity for $2 \mathrm{~h}$ to allow the cells in suspension to adhere to the PDMS pedestal.

15. Aspirate the drop of medium, removing the unattached cells. Wash with warm PBS and fill the wells with warm culture medium.

16. Once the cells have filled the support they will begin to migrate onto the wires and the 6-well plate is ready to be put under the microscope for imaging (Fig. 2B).

\subsection{Wavy Substrates (Fig. 1B)}

1. The pattern on the mask is transferred to a thick SU8 negative resist (the example is for SU82150 , ending up in a castellated substrate where the patterns are about $200 \mu \mathrm{m}$ high) (see Note 36):

a. Spin coat the resist on a silicon wafer (3000 rpm, 30s)

b. Soft bake $40 \mathrm{~min}, 95^{\circ} \mathrm{C}$. Allow to cool down to room temperature.

c. Expose through the mask with the mask aligner.

d. Post exposure bake: $15 \mathrm{~min}, 95^{\circ} \mathrm{C}$. Allow to cool down to room temperature.

e. Develop in SU8 developer: 15 min. Rinse and dry.

2. Coat with fluorinated silane: place a few drops of fluorosilane in a small polyethylene weighting boat in a vacuum desiccator. Plasma treat the castellated SU8 structure $(1 \mathrm{~min})$ and put the activated pattern in the desiccator. Apply vacuum for typically $2 \mathrm{~h}$ (see Note 37).

3. Pour $5 \mathrm{~g}$ of the freshly prepared PDMS mix over the master. Degas and cure overnight at $65^{\circ} \mathrm{C}$. Allow to cool down and unmold.

4. Place $2.8 \mathrm{~g}$ of polystyrene granules in the heating press and inside a square spacer $(3 \mathrm{~cm} * 3 \mathrm{~cm}$; height $=3 \mathrm{~mm}$ ). The spacer sets the thickness of the polystyrene plate and acts as a frame to confine lateral dimensions. Heat the polystyrene at $150^{\circ} \mathrm{C}$ in the press for $10 \mathrm{~min}$ between two smooth metal plates to yield a flat polystyrene plate. Cool down to ambient temperature.

5. Melt the flat PS plate under the same experimental conditions $\left(150^{\circ} \mathrm{C}, 4\right.$ bars), intercalating the PDMS mold between one of the plates and the PS, to emboss the periodic castellated shape into 
the softened PS. Allow to cool down and unmold the PDMS master.

6. Leave the PS substrate at $80^{\circ} \mathrm{C}$ (below the glass transition temperature) under vacuum overnight to pump out gas and moisture contained inside that may otherwise deform the substrate during subsequent high temperature reflow.

7. Reflow the PS plate under vacuum at $180^{\circ} \mathrm{C}$, for 50 to $120 \mathrm{~min}$ (see Notes 38 and 39) in order to modulate the wavy shape and therefore the out-of-plane curvature of the substrate. Quench to room temperature (Fig. 1C).

8. Duplicate the wavy PS substrate in PDMS (see step 3) (see Note 40).

9. Plasma oxidize the PDMS surface for $30 \mathrm{~s}$ with air plasma and incubate with fibronectin solution for $1 \mathrm{~h}$ before culturing cells.

10. Incubate the cells at appropriate temperature and humidity for $30 \mathrm{~min}$ to allow them to adhere to the substrate.

11. Aspirate medium with unattached cells. Wash with warm PBS and fill with warm culture medium. Cell growth and migration can now be observed (Fig. 2C).

\section{NOTES}

1. Prepare immediately before use.

2. Use low molecular weight polymer : average $M_{n} 950$

3. Wear UV protective glasses when using UV radiations

4. A collimated UV lamp of sufficient power at $365 \mathrm{~nm}$ can also be used.

5. The Chrome features on the mask correspond to the non-adherent moiety.

6. It is mandatory that the mask is transparent for wavelengths down to $185 \mathrm{~nm}$

7. The wavelength has to be less than $200 \mathrm{~nm}$.

8. These lamps generate ozone that is a toxic and irritant gas. Use under a fume hood or hook to an 'ozone killer'. 
9. Use glass Pasteur pipettes to produce capillaries whose radius is larger than $20 \mu \mathrm{m}$, by heating and pulling

10. Use these glass rods with the pipette puller. Alternatively $40 \mu \mathrm{m}, 60 \mu \mathrm{m}$ and $85 \mu \mathrm{m}$ diameter glass wires can be bought from CM Scientific and used as received.

11. If very small diameters are needed.

12. Note that we use a negative photoresist and therefore the Chrome features on the mask correspond to the valleys in the final PDMS substrate.

13. Other motifs can also be used such as arrays of disks.

14. Caution! Very exothermic when mixing. Prepare in small quantities. Add the $\mathrm{H}_{2} \mathrm{O}_{2}$ slowly in the acid. Wear reinforced gloves and face shield. Dispose appropriately.

15. At this point, both sides of the coverslips should be hydrophobic.

16. Use a sonic bath to dissolve the products.

17. The coverslips should be well separated.

18. ... or until a white halo is formed between the coverslip and the glass Petri dish.

19. The UV light comes from the bottom and illuminates the slides through the glass bottom of the Petri dish.

20. The grafting reaction occurs preferentially between the coverslips and the Petri dish bottom, presumably because of the slow diffusion of oxygen in this confined area.

21. Now, the grafted side is hydrophilic while the ungrafted silanized side is still hydrophobic. You can test which side is grafted by its wetting properties using a DI water droplet.

22. From this step on, only one side of each coverslip is coated. Keep track of which one.

23. Timing here is critical. Use the same time as acrylamide step as a starting point.

24. Up to development, photoresist is to be handled only in inactinic light.

25. If resolution is not an issue, keep the temperature as low as possible. We had satisfactory results with $\mathrm{T}=80^{\circ} \mathrm{C}$.

26. The exposure time depends on the lamp power. In doubt, follow manufacturer instructions. 
27. Do not let the coverslips in the plasma for more than $5 \mathrm{~min}$ in one run because the heat will degrade the protective photoresist and consequently the underlying cell-repellent layer.

28. The acetone treatment does not affect the fibronectin coating as can be checked by culturing cells on these substrates.

29. 30 min on Fibronectin-coated substrates

30. Be careful not to scratch the repellent layer with the tweezers.

31. Chrome side appears darker and less shiny than the glass side

32. Avoid bubbles as they will affect the transmission of the UV light and deform the patterns.

33. Add more DI water to facilitate the process.

34. Pull sharply when the glass becomes soft over the flame.

35. Around 2-5 $10^{6}$ cells $/ \mathrm{mL}$.

36. For more details, refer to manufacturer instructions.

37. This procedure makes it easier to mold the structure with PDMS by decreasing the friction between the two polymers.

38. Vacuum is used to prevent polymer degradation

39. Adjust reflow time and temperature to attain the exact curvature profile that is needed. Measure the profile with a stylus profilometer.

40. The PS wavy substrate is used as a master to mold as many PDMS substrates as necessary. 


\section{Figures}

Fig. 1: A Preparation of confining patterns. The two possible methods are photolithography or deep UV patterning. B Fabrication of wavy substrates. The castellated substrate structured with thick photoresist is duplicated in PDMS and then embossed in molten PS. The PS structure is then reflowed at high temperature. The resulting wavy pattern in PS can be duplicated indefinitely in PDMS. C Profiles of the PS mold after $45 \mathrm{~min}$ (red) and $1 \mathrm{~h}$ (black) reflow at $180^{\circ} \mathrm{C}$ (the baseline has been subtracted).

Fig. 2: A Collective displacements of MDCK cells in a confining disk. The velocity field (red) obtained by PIV is superimposed to the phase contrast image. B MDCK monolayer cultured on a $100 \mu \mathrm{m}$ diameter cylindrical glass fiber. Only the top half is represented (Blue: nuclei; green: actin). C MDCK monolayer on a wavy surface (red nuclei). The period of the pattern is $90 \mu \mathrm{m}$. 


\section{ACKOWLEDGMENTS}

We gratefully acknowledge financial support from the Groupement des Entreprises Françaises dans la Lutte contre le Cancer (GEFLUC), the Région Ile-de-France Domaine d'Intérêt Majeur (DIM) Nano-K, the Association pour la Recherche sur le Cancer (ARC), the EU cofund PRESTIGE post-doc program, the EU cofund IC-3i PhD program, and the Fondation Pierre-Gilles de Gennes. The "Biology-Inspired Physics at MesoScales" group is member of the CelTisPhyBio Labex and of the Institut Pierre-Gilles de Gennes. It is a pleasure to thank Mohamed El Beheiry for his help in the 3D processing of our images.

\section{REFERENCES}

1. E. Scarpa and R. Mayor (2016) Collective cell migration in development, J. Cell Biol. 212, $143-155$.

2. P. Rørth (2012) Fellow travellers: emergent properties of collective cell migration., EMBO Rep. 13, 984-991.

3. V. Hakim and P. Silberzan (2017) Collective cell migration : a physics perspective, Reports Prog. Phys. in press,.

4. N.S. Gov (2014) Collective Cell Migration, In: Kaunas, R. and Zemel, A. (eds.) Cell and Matrix Mechanics, pp. 219-238 CRC Press, Boca Raton.

5. B. Weigelin, G.-J. Bakker, and P. Friedl (2012) Intravital third harmonic generation microscopy of collective melanoma cell invasion, IntraVital. 1, 32-43.

6. S.R.K. Vedula, M.C. Leong, T.L. Lai, et al. (2012) Emerging modes of collective cell migration induced by geometrical constraints., Proc. Natl. Acad. Sci. U. S. A. 109, 12974-9.

7. H.G. Yevick, G. Duclos, I. Bonnet, et al. (2015) Architecture and migration of an epithelium on a cylindrical wire, Proc. Natl. Acad. Sci. 112, 5944-5949. 
8. Y. Zheng, J. Chen, M. Craven, et al. (2012) In vitro microvessels for the study of angiogenesis and thrombosis, Proc. Natl. Acad. Sci. 109, 9342-9347.

9. M. Ye, H.M. Sanchez, M. Hultz, et al. (2014) Brain microvascular endothelial cells resist elongation due to curvature and shear stress, Sci. Rep. 4, 4681.

10. A. Tourovskaia, T. Barber, B.T. Wickes, et al. (2003) Micropatterns of Chemisorbed Cell Adhesion-Repellent Films Using Oxygen Plasma Etching and Elastomeric Masks, Langmuir. $19,4754-4764$.

11. A. Tourovskaia, X. Figueroa-Masot, and A. Folch (2006) Long-term microfluidic cultures of myotube microarrays for high-throughput focal stimulation., Nat. Protoc. 1, 1092-104.

12. M. Deforet, V. Hakim, H.G. Yevick, et al. (2014) Emergence of collective modes and tridimensional structures from epithelial confinement., Nat. Commun. 5, 3747.

13. V. Nier, M. Deforet, G. Duclos, et al. (2015) Tissue fusion over nonadhering surfaces, Proc. Natl. Acad. Sci. 112, 9546-9551.

14. G. Duclos, C. Erlenkämper, J.-F. Joanny, et al. (2017) Topological defects in confined populations of spindle-shaped cells, Nat. Phys. 13, 58-62.

15. A. Azioune, N. Carpi, Q. Tseng, et al. (2010) Protein Micropatterns. A Direct Printing Protocol Using Deep UVs., Methods Cell Biol. 97, 133-146.

16. M. Thery and M. Piel (2014) Scientific Protocols - Adhesive Micropatterns for Cells: A Microcontact Printing Protocol, Sci. Protoc. DOI 10.5281/zenodo.13592.

17. A. Azioune, M. Storch, M. Bornens, et al. (2009) Simple and rapid process for single cell micro-patterning., Lab Chip. 9, 1640-1642.

18. G. Duclos, S. Garcia, H.G. Yevick, et al. (2014) Perfect nematic order in confined monolayers of spindle-shaped cells, Soft Matter. 10, 2346-2353. 
